\title{
3 Elaboración del mapa de ruido de las calles de la ciudad de Cuenca, a partir de las características viales y la densidad del tráfico
}

Tacuri Ortega, Christian' ; Calderón Peralvo, Felipe ${ }^{2}$; Martínez Gavilanes, Julia3; Sellers Walden, Chester ${ }^{4}$; Delgado Inga, Omar

Ingeniero en Mecánica Automotriz por la Universidad del Azuay, maestrante de Gestión de Mantenimiento en la Universidad del Azuay, investigador en el IERSE de la Universidad del Azuay en el área de monitoreo del ruido ambiente generado por el tránsito vehicular en la ciudad de Cuenca.

christian.t.o@hotmail.com

Ingeniero en Mecánica Automotriz por la Universidad del Azuay, investigador en el IERSE de la Universidad del Azuay en el área de monitoreo del ruido ambiente generado por el tránsito vehicular en la ciudad de Cuenca.

Ingeniera Civil por la Universidad de Cuenca, Especialista en sistemas de información geográfica, aplicados a la gestión territorial y ambiental por la Universidad del Azuay, Master en Desarrollo Local con mención en formulación y evaluación de proyectos de desarrollo endógeno por la Universidad Politécnica Salesiana sede Cuenca. Docente e Investigadora en el IERSE de la Universidad del Azuay.

Ingeniero de Sistemas por la Universidad del Azuay, Master Universitario en Gestión Sostenible de la Tierra y Territorio por la Universidad Santiago de Compostela, Magister en Geomática con mención en Ordenamiento Territorial por la Universidad del Azuay. Docente e Investigador en el IERSE de la Universidad del Azuay.

Ingeniero en Minas por la Universidad del Azuay, Master Universitario en Tecnologías de la Información Geográfica por la Universidad Alcalá de Henares, Especialista en sistemas de información geográfica aplicados a la gestión territorial y ambiental por la Universidad del Azuay. Docente e Investigador en el IERSE de la Universidad del Azuay.

\section{Christian \\ Tacuri \\ Ortega ${ }^{1}$}

\section{Julia \\ Martínez Gavilanes $^{3}$}

Omar
Delgado
Inga 


\section{Resumen}

Debido al progresivo crecimiento del parque automotor en la ciudad de Cuenca-Ecuador, se evidencian congestiones por la sobresaturación del tráfico en las vías de la ciudad, las cuales colapsan en horas pico, ocasionando, como consecuencia, elevados niveles de ruido. Por esta razón, el presente artículo tiene como objeto modelar el ruido generado por el tráfico vehicular en las principales calles de la ciudad. Para ello se utilizará el modelo de predicción de ruido NMPB-Routes-96 en el software especializado en ruido "Datakustik CadnaA"; para el efecto se ha realizado un levantamiento de los datos requeridos por el software, además de validar, depurar y sistematizar la información de la geodatabase proporcionada por la Dirección Municipal de Tránsito y Transporte (DMT) del GAD municipal de Cuenca, en la cual hay información sobre las características viales y del parque automotor de la ciudad.

\section{Palabras clave:}

Ruido, mapa, CadnaA, nivel de presión sonora.

\begin{abstract}
Due to the progressive growth of the car park in the city of Cuenca-Ecuador, there is evidence of congestion due to the over-saturation of traffic on the city's roads, which collapse in peak hours, resulting in high noise levels, resulting in high noise levels. For this reason, this article aims to model the noise generated by vehicular traffic in the main streets of the city, using the noise prediction model NMPB-Routes-96 in the specialized noise software "Datakustik
\end{abstract}

CadnaA", for this purpose a survey of the data required by the software has been carried out, besides validating, debugging and systematizing of the geodatabase information provided by the Municipal Transit and Transport Department (DMT) of the municipal GAD of Cuenca, in which there is information about the road characteristics and of the automotive park of the city.

\section{Keywords:}

Noise, map, CadnaA, sound pressure level. 


\section{Introducción}

La contaminación acústica en las zonas urbanas constituye un problema ambiental que ha acaparado el interés de investigadores, organismos de control ambiental y de los ciudadanos, debido a que, desde hace algunos años, se ha considerado como uno de los factores que más influye en el deterioro de la calidad de vida, consecuencia de la falta de estrategias y de políticas para su control.

Según la Organización Mundial de la Salud (OMS) existe una relación directa y exponencial entre el nivel de desarrollo de un país y el grado de contaminación acústica que impacta a su población (Ramírez González \& Domínguez Calle, 2011). En vista de que este problema afecta a la salud y a la calidad de vida de la población, se han desarrollado mapas de ruido en: España, México, Brasil, Colombia, Chile, entre otros países, los que han utilizado el software Datakustik CadnaA para su realización, el cual sirve para caracterizar el grado de contaminación sonora y así establecer planes de acción para mitigar dicho problema.

La investigación de las emisiones sonoras ha permitido la elaboración de mapas de ruido del área urbana de la ciudad de Cuenca, con el objetivo de contar con información que nos permita determinar los cambios y la dinámica que ha tenido el ruido en la ciudad y con base en esta información proponer estrategias para mitigar la contaminación acústica. Los mapas de ruido se han elaborado con base en las investigaciones del comportamiento de las emisiones sonoras con información correspondiente a los años 2012 y 2014.

En este proyecto, con el fin de continuar con la elaboración de mapas de ruido de la ciudad, se realizó una revisión, validación y sistematización de la base de datos proporcionada por el GAD municipal de Cuenca. Además se estableció una metodología para la realización de mapas de ruido mediante el software especializado Datakustik CadnaA, para de esta manera proceder con la elaboración del mapa de ruido del año 2015.

\section{Objetivos Objetivo general}

Validar y sistematizar la información de la geodatabase proporcionada por la Dirección Municipal de Tránsito y Transporte (DMT) del GAD Municipal de Cuenca, para elaborar el mapa de ruido al 2015, de la zona urbana de la ciudad de Cuenca, mediante el software Datakustik CadnaA.

\section{Objetivos específicos:}

a) Levantamiento de información requerida por el software CadnaA.

b) Revisión, validación, depuración y sistematización de la información recopilada en una geodatabase.

c) Elaboración del mapa de ruido al año 2015, con el software de ruido CadnaA.

\section{Metodología}

La metodología utilizada para la ejecución de este proyecto es la siguiente:

1. Parte de la revisión, validación y depuración de la base de datos entregada por la DMT.

2. Determinación de la información faltante, luego se procede a realizar el levantamiento de datos correspondiente.

3. Se sistematiza la información de predios y manzanas de la ciudad de Cuenca. 
4. Simultáneamente se configura el software CadnaA para la parte de cálculo, la de objetos y la malla de receptores.

5. Con la base de datos previamente sistematizada y el software configurado se elabora el modelamiento del ruido en el área urbana de la ciudad de cuenca.

6. Se utilizan las coordenadas de los puntos de monitoreo de proyectos anteriores para realizar la comparación correspondiente con los niveles permitidos en la legislación nacional TULSMA.

\section{Recopilación y levantamiento de datos}

En primer lugar se analizó toda la información disponible, partiendo de la base vial en formato shapefile suministrada por la Dirección Municipal de Tránsito (DMT), de la cual se utilizan datos como: intensidad media diaria (IMD), material de la calzada, tipo de vía, ancho de vía, número de carriles y velocidad máxima de circulación.

Esta base fue recortada a los límites de la zona de estudio, es decir, se mantienen únicamente las vías que cuentan con datos de IMD, que se encuentran dentro del perímetro urbano de la ciudad de Cuenca.

A continuación se detallan las acciones en los parámetros a utilizar en el cálculo del mapa de ruido.

\section{Intensidad media diaria (IMD)}

La intensidad media diaria (IMD), según Bañón Blázquez, se define como el número total de vehículos que atraviesan una determinada sección durante un año, dividido por 365 (Bañón Blázquez, 2000).
Al verificar los datos de IMD se detectaron ciertas inconsistencias, las mismas que fueron corregidas, de manera que no se modificara demasiado la base de datos, para esto se localizaron solo aquellas que presentan una variación mayor al 15\% y se analizaron estos casos de manera individual, con base en los siguientes criterios de corrección:

- Igualar el valor de IMD de un tramo intermedio cuando los tramos anterior y posterior tienen el mismo dato.

- Cuando un dato de IMD es muy bajo respecto del siguiente, se realiza un promedio entre dichos datos y el valor obtenido lo asume el dato inferior.

- En los casos donde existe una bifurcación, se divide el dato anterior a ésta y se coloca en cada vía.

Dichos criterios fueron puestos en consideración de la DMT, que aprobó la normalización de la base de datos para la realización de este proyecto.

\section{Material de la calzada}

En cuanto al material de la calzada, para el caso de la Av. 12 de abril (sector Universidad de Cuenca), se consideró como dos vías por separado, debido a que el material de la calzada en el carril con sentido de circulación Norte-Sur es adoquín, mientras que el carril Sur-Norte es de hormigón. Para esto además se dividió el valor del IMD para dos, asignando este resultado a cada vía.

Además, según las opciones disponibles en el software CadnaA se determinó utilizar los siguientes materiales de calzada:

- Adoquín = Empedrado en población

- Lastre = Empedrado en población 


$$
\begin{array}{ll}
\text { - Asfalto } & \text { Asfalto poroso } \\
\text { - Hormigón } & =\text { Asfalto liso }
\end{array}
$$

\section{Tipo de vía}

En este apartado, según las opciones disponibles en el software CadnaA, se determinó los tipos de vía a utilizar:

$$
\begin{array}{lll}
- & \text { Calle } & =\text { Vía local } \\
\text { - } & \text { Avenida } & =\text { Carretera principal } \\
\text { - } & \text { Autopista } & =\text { Autopista }
\end{array}
$$

\section{Distancia entre las líneas centrales de los carriles exteriores}

El parámetro de distancia entre las líneas centrales de los carriles exteriores, requerido por el software CadnaA, se obtuvo a partir del ancho de vía y número de carriles, con las siguientes consideraciones:

- Para vías con un solo carril ingresar el valor de cero debido a que debe existir una sola línea de emisión, la cual pasa por el centro de la vía.

- Para vías de dos o más carriles, se divide el ancho de la vía para el número de carriles y luego este valor se resta del ancho de la vía.

Además, se determinó que las avenidas Solano, 10 de Agosto (sector Virgen de Bronce) y 24 de Mayo (sector Gapal), que cuentan con un parterre muy amplio o variable, deben considerarse como vías por separado, y el dato de IMD dividirse al igual que en el caso de la Av. 12 de Abril.

Velocidad máxima de circulación

La velocidad máxima de circulación fue actualizada según los datos establecidos en la propuesta del Plan de Movilidad y Espacios Públicos (2015-2025) del GAD Cuenca (Municipalidad de Cuenca, 2015).

\section{Tipo de flujo de circulación}

Según el Diario Oficial de la Unión Europea, para seleccionar este campo, se deben utilizar las siguientes consideraciones:

- Tráfico fluido: El flujo es estable en tiempo y espacio, en períodos de 10 minutos. Este tipo de flujo se aplica en autopistas, autovías, carreteras interurbanas y vías rápidas.

- Tráfico intermitente: Flujo de vehículos en transición, es decir, tienen variaciones bruscas del flujo en períodos de tiempo cortos. Este tipo de flujo se aplica para calles de centros urbanos, vías importantes próximas a saturación, vías con numerosas intersecciones, etc.

- Aceleración: Es un flujo en pulsos, es decir que una proporción significativa de vehículos está acelerando. Este tipo de flujo se observa en vías rápidas después de una intersección o al salir de peajes.

- Desaceleración: Es el flujo contrario al anterior, una proporción importante de vehículos está desacelerando. Este tipo de flujo se observa en grandes intersecciones urbanas, en salidas de autopistas y en vías rápidas en aproximaciones a peajes (Diario Oficial de la Unión Europea, 2003).

Considerando lo anterior no se pueden utilizar los parámetros de aceleración y desaceleración, debido a que no se cuenta con la estructura vial adecuada (shapefile), es decir, no se tienen vías con sentidos de circulación por separado, por lo cual se estableció utilizar el parámetro de tráfico intermitente para todas las vías, excepto para aquellas que permiten un flujo continuo, como es el caso de la circunvalación sur, panamericana norte, panamericana sur, vía a 
Huishil y los caminos a Ochoa León, Paccha, Ricaurte, Mayancela, Sinincay y Turi.

Luego del filtrado, organizado, corregido y completado todo el conjunto de parámetros requeridos para el cálculo del mapa de ruido en una geodatabase, es necesario modificar el título de los campos según la tabla de atributos y abreviaciones del software CadnaA para su correcta importación de la información, como se indica en la tabla 1.

Tabla 1. Tabla de atributos y abreviaciones de los datos utilizados.

\begin{tabular}{|c|c|}
\hline \multicolumn{1}{|c|}{ CAMPO } & ABREVIATURA \\
\hline $\begin{array}{c}\text { Nombre de vía } \\
\text { Intensidad media diaria } \\
\text { (IMD) }\end{array}$ & BEZ \\
\hline $\begin{array}{c}\text { Material de la calzada } \\
\text { Tipo de vía }\end{array}$ & DTV \\
\hline $\begin{array}{c}\text { Distancia entre las líneas } \\
\text { centrales de los carriles } \\
\text { exteriores }\end{array}$ & STRGATTNR \\
\hline $\begin{array}{c}\text { Velocidad máxima } \\
\text { Tipo de flujo }\end{array}$ & RPKW \\
\hline $\begin{array}{c}\text { Tipo de altura (relativa o } \\
\text { absoluta) }\end{array}$ & FLOWNR \\
\hline
\end{tabular}

Fuente: (DataKustik GmbH, 2004).

En la cartografía de edificaciones, debido a que el software posee una restricción de cálculo únicamente a 1.000 objetos, se decidió utilizar un archivo shapefile de manzanas de la zona urbana de Cuenca del que se obtiene un resultado similar al de la técnica utilizada en el estudio de Nicolás Bastián, donde se realizan agrupaciones de edificios para reducir la cantidad de objetos. Sin embargo, este archivo tiene alrede- dor de 5000 edificaciones, lo cual también sobrepasa el límite máximo de objetos a importar al software CadnaA, por lo que fue necesario utilizar métodos de simplificación para obtener el mapa de ruido de toda la ciudad (Bastián, 2015).

Para simplificar el número de objetos a menos de 1000, se consideró la agrupación de estos mediante polígonos, de tal manera que dicha acción no obstaculice las entradas aledañas a las vías de estudio para que el ruido ingrese normalmente, lo que permitió realizar el cálculo y representación del mapa de ruido de la zona urbana de la ciudad. Además, se realizaron pruebas comparativas en el software CadnaA utilizando este método y el de manzanas separadas, en donde se colocaron receptores en varios puntos de monitoreo, con lo cual se determinó que prácticamente no existe variación en los niveles de presión sonora equivalente (NPSeq).

En cuanto a la altura de los objetos, se consideró una altura promedio por manzanas, tomando en cuenta que, según la arquitectura de la ciudad, la mayoría de edificaciones son de dos pisos y cada piso tiene una altura de tres metros, con lo que se establecieron tres criterios en el levantamiento de información:

- 6 metros para las manzanas que contengan la mayor parte de sus edificaciones de 2 pisos.

- 12 metros para aquellas que contengan la mayor parte de sus edificaciones de 3 pisos en adelante.

- 20 metros para edificaciones puntuales que tengan gran altura, como el caso de edificios departamentales, etc. 


\section{Procesamiento de datos}

El mapa de ruido se elaboró mediante modelación, utilizando el software Datakustik CadnaA versión 3.5.11. Este software permite predecir y evaluar el ruido ambiental de fuentes emisoras como industriales, ferroviarias, vehiculares, entre otras. El proceso de modelación se hizo alimentando el programa con la información requerida, que corresponde principalmente a información cartográfica y de aforo vehicular (Bastián, 2015).

El software tiene varios modelos de cálculo, los cuales se utilizan de acuerdo con la fuente emisora de ruido. Para este proyecto, al considerar solo el tráfico rodado como fuente de ruido, se utilizó el modelo francés NMPB-Ruotes-96, porque es el modelo de predicción del nivel de presión sonora equivalente (NPSeq) más difundido, además de ser el recomendado por la Unión Europea en la directiva 2002/49/CE.

\section{Configuración de cálculo}

El software CadnaA requiere de la configuración de ciertos parámetros que definen las características y el tipo de ruido a ser analizado, que para este caso es el ruido provocado por el tráfico vehicular. Entre los parámetros principales para la realización del mapa de ruido de la ciudad de Cuenca, están el aforo vehicular y la infraestructura vial por el ancho de vía y tipo de calzada.

Dentro de las configuraciones necesarias está el modelo de cálculo a utilizar, que es el modelo general establecido por la Unión Europea, denominado "UE Interim", en el cual se aplican las normas de cálculo para el apartado de carreteras, de acuerdo con el modelo francés NMPB-Routes-96. Además se definieron los límites de cálcu- lo para el mapa de ruido, entre los cuales figuran el radio de búsqueda de objetos de $1000 \mathrm{~m}$, radio de búsqueda de fuentes de $100 \mathrm{~m}$, distancia máxima entre fuente - receptor de $1000 \mathrm{~m}$, así como considerar únicamente el primer orden de reflexión y la interpolación de la malla de receptores de $17 \times 17$ metros con el fin de optimizar y minimizar el tiempo de cálculo.

Por otra parte, es necesario configurar los objetos que se utilizan en el mapa de ruido, como las vías que contienen información de aforo vehicular e infraestructura vial. Los edificios se configuraron con la opción de reflectividad de ruido "Sin reflexión" puesto que en estudios previos se determinó que era la configuración que proporcionó los niveles de presión sonora más equiparables con datos obtenidos con sonómetro; y los receptores, los cuales se configuraron según la norma nacional TULSMA.

\section{Resultados}

Como se observa en las figuras 1 y 2 , se obtuvo un mapa de ruido de las calles de la zona urbana de Cuenca, tanto para el día como para la noche, en el que se puede diferenciar claramente aquellas zonas más afectadas por los elevados niveles de presión sonora, gracias a una clara escala de colores y la debida referenciación de la ciudad.

Según la clasificación de los usos de suelo de la norma TULSMA, la zona comercial registró las máximas emisiones de ruido, seguida de la zona residencial, como la zona de equipamiento de servicios sociales. Todas ellas superan los límites permisibles de la legislación nacional; en cambio, la zona industrial registra emisiones inferiores al límite permisible. 
Como se puede observar en el mapa de ruido de la figura 1, las vías de mayor aforo vehicular presentan mayores niveles de presión sonora. Estas vías se presentan con una coloración azul oscuro, que representa niveles entre 75 dBA y 80 dBA. Entre las principales vías de emisión de ruido es- tán la Av. de las Américas, la circunvalación sur, la Doce de Abril, la Solano y vías del centro histórico de la ciudad, mientras que vías de menor flujo de tráfico presentan niveles inferiores de ruido, que se muestra con una coloración morada, que corresponde a 70 dBA aproximadamente.

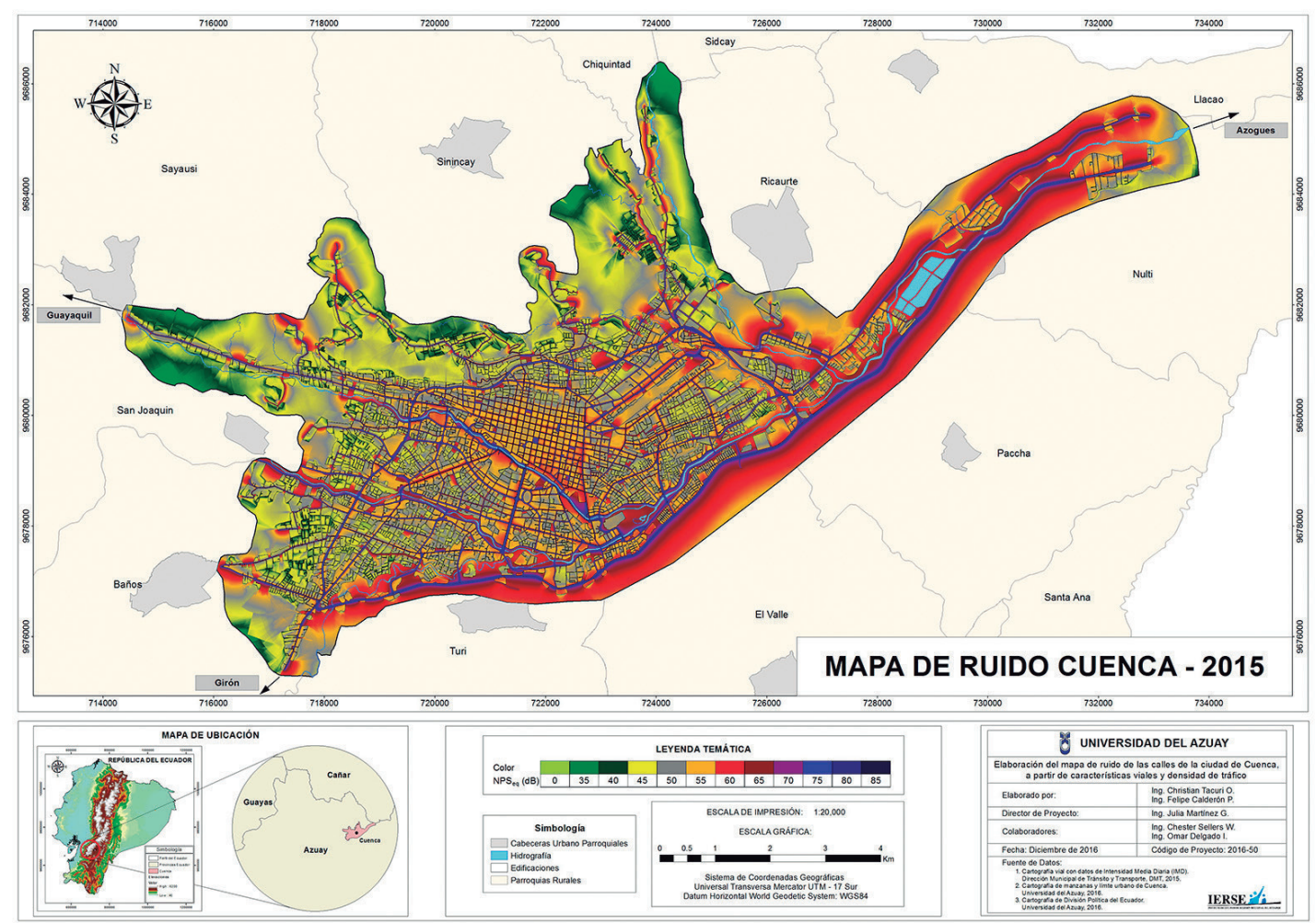

Figura 1. Mapa del ruido diurno de la ciudad de Cuenca, año 2015 


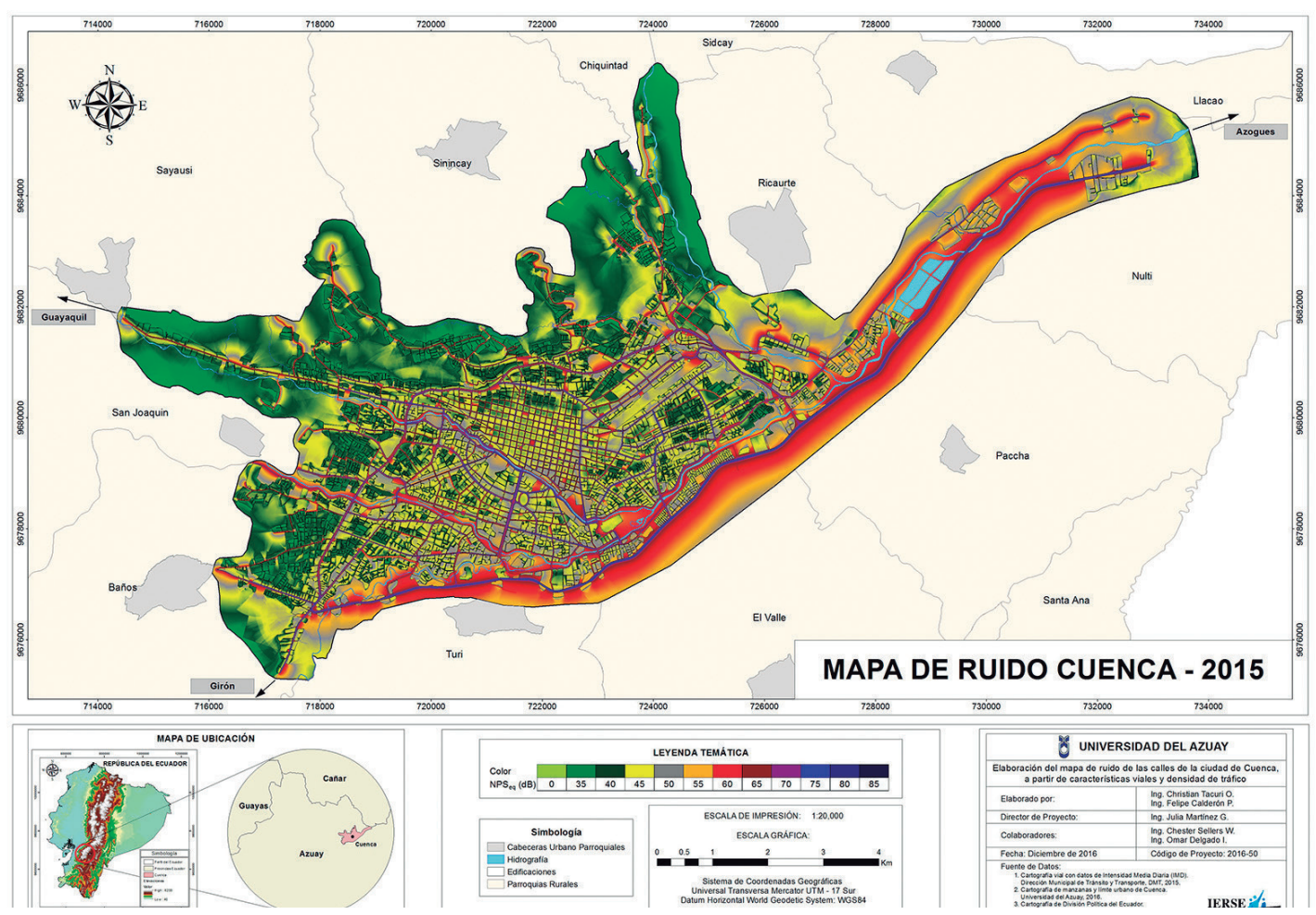

Figura 2. Mapa del ruido nocturno de la ciudad de Cuenca, año 2015

\section{Conclusiones}

La representación del mapa de ruido de las calles de la ciudad de Cuenca, obtenido a través de modelación con el software CadnaA, resultó satisfactorio, ya que permitió diferenciar claramente las vías que emiten mayores niveles de ruido, debido a su cantidad de tráfico, además del elevado número de vehículos pesados que circulan por las mismas. Esto permitió detectar los lugares más afectados de la ciudad y en base a esto proponer alternativas para mitigar dicho problema.

Como se puede observar en el mapa del ruido, los niveles de presión sonora se concentran en las vías, puesto que por el tráfico vehicular son la fuente de emisión de ruido, en tanto que la superficie que ocupan las edificaciones registra niveles inferiores, debido a que la propagación del ruido disminuye por la reflectividad de las fachadas, es decir, mientras más apartado se encuentre un objeto de la vía, menor será el nivel de presión sonora registrado en el mapa del ruido.

De los resultados obtenidos en el mapa se concluye que, de los 26 puntos analizados, únicamente los correspondientes a la zona industrial se encuentran por debajo de los límites máximos permisibles, lo que representa el 11.5\%; en tanto que el 88.5\% restante está sobre los límites establecidos en el TULSMA. 
Los diez puntos evaluados en la zona comercial presentan niveles de ruido que se encuentran sobre los límites máximos permisibles, siendo la bajada del centenario la más crítica, ya que en este se registraron los valores de ruido más altos. De igual manera, para la zona residencial los diez puntos evaluados están sobre los límites establecidos en el TULSMA, siendo el sector de Challuabamba en donde se obtuvieron los NPSeq más elevados.

En la zona de equipamiento de servicios sociales se tomaron tres puntos de muestreo, y ninguno de ellos presentó niveles de ruido dentro de los límites establecidos en la norma para el período diurno y nocturno.

\section{Recomendaciones}

- En vista de que en la mayoría de las zonas clasificadas según los usos del suelo que establece el TULSMA, se superan los límites establecidos, se recomienda implementar políticas para mejorar el sistema de movilidad y transporte de la ciudad y a disminuir la presión sonora.

- Realizar campañas para difundir e incentivar el uso de medios de transporte público haciendo énfasis en el uso de medios de transporte alternativo, como por ejemplo las bicicletas, ya que en la ciudad se han construido ciclovías y rutas recreativas, que no tienen el uso para el que fueron construidas.

- Promocionar desde el uso del medio de transporte masivo "tranvía 4 ríos" para que cuando entre en servicio la ciudadanía tome en conciencia y empiece a apoyar este nuevo sistema de transporte.

- Informar a la ciudadanía los resultados de este proyecto para que se conozca la situación actual del ruido que se produce en la ciudad y que de una u otra manera traten de colaborar con el fin de mitigar este problema que nos afecta a todos los habitantes de la ciudad. 


\section{Referencias}

Bañón Blázquez, L. (2000). Manual de carreteras. Alicante: Ortiz e Hijos, Contratista de Obras, S.A. Retrieved from http://sirio.ua.es/proyectos/ manual_ carreteras/01020103.pdf

Bastián, N. (2015). Elaboración de mapa de ruido de la ciudad de Valdivia mediante software de modelación utilizando métodos de simplificación. Síntesis Tecnológica,(Octubre2015),14. Retrieved from https://www.researchgate.net/ publication/282914265 Elaboracion de_mapa_de_ruido_de_la_ciudad_ de_Valdivia_mediante_software_de_ modelacion_utilizando_metodos_de_ simplificacion

DataKustik GmbH. (2004). CadnaA. Greifenberg.

Diario Oficial de la Unión Europea. (2003). Recomendación de la Comisión de 6 de agosto de 2003 relativa a las Orientaciones sobre los métodos de cálculo provisionales revisados para el ruido industrial, procedente de aeronaves, del tráfico rodado y ferroviario, y los datos de emisiones correspondientes. Ministerio de La Presidencia de España. Retrieved from https://www.boe.es/doue/2003/212/ L00049-00064.pdf

Municipalidad de Cuenca. (2015). Plan de movilidad y espacios públicos (Tomo II). Cuenca. Retrieved from http:// www.cuenca.gob.ec/?q=system/files/ PMEPCUENCA2015_tomo_II.pdf

Ramírez González, A., \& Domínguez Calle, E. A. (2011, December). El ruido vehicular urbano: Problemática agobiante de los países en vías de desarrollo. Revista de la Academia Colombiana de Ciencias Exactas, Físicas y Naturales, 35(137), 509-530. Retrieved from http://www.scielo.org.co/scielo. php? script=sci_arttext\&pid=S037039082011000400009\&lng=en\&nrm=iso \&tlng=es 